Draft Version OCtober 20, 2018

Preprint typeset using $\mathrm{IATE}_{\mathrm{E}} \mathrm{X}$ style emulateapj v. 08/22/09

\title{
RIDING THE SPIRAL WAVES: IMPLICATIONS OF STELLAR MIGRATION FOR THE PROPERTIES OF GALACTIC DISKS
}

\author{
Rok Roškar $^{1}$, Victor P. Debattista ${ }^{2}$, Thomas R. Quinn ${ }^{1}$, Gregory S. Stinson ${ }^{3}$, James WAdsley $^{3}$ \\ Draft version October 20, 2018
}

\begin{abstract}
Stars in disks of spiral galaxies are usually assumed to remain roughly at their birth radii. This assumption is built into decades of modelling of the evolution of stellar populations in our own Galaxy and in external systems. We present results from self-consistent high-resolution $N$-body + Smooth Particle Hydrodynamics simulations of disk formation, in which stars migrate across significant galactocentric distances due to resonant scattering with transient spiral arms, while preserving their circular orbits. We investigate the implications of such migrations for observed stellar populations. Radial migration provides an explanation for the observed flatness and spread in the age-metallicity relation and the relative lack of metal poor stars in the solar neighborhood. The presence of radial migration also prompts rethinking of interpretations of extra-galactic stellar population data, especially for determinations of star formation histories.

Subject headings: galaxies: evolution — galaxies: spiral — galaxies: stellar content — Galaxy: solar neighborhood - Galaxy: stellar content — stellar dynamics
\end{abstract}

\section{INTRODUCTION}

In a universe where mass assembles by accretion of progressively larger constituents, thin disks of galaxies form during quiescent evolution following the major merging epoch (Brook et al. 2004; Robertson et al. 2006). The inner parts, with lower angular momentum assemble first, followed by the higher angular momentum components, resulting in "inside-out" build up of disk material (Larson 1976; White \& Frenk 1991; Muñoz-Mateos et al. 2007). Because gas can efficiently dissipate energy, its motion around the galaxy is mostly circular. Stars born in such gas disks are initially on nearly circular orbits, but their infant kinematic state is highly fragile; nonaxisymetric perturbations such as bars or spiral arms readily drive the orbits away from circular. Despite the increase in eccentricity, mean orbital radii are assumed to remain relatively constant. As a result, interpretations of Galactic and extra-galactic stellar population observations invariably make the fundamental assumption that stars remain at roughly their birth radii throughout their lives. Consequently, this assumption is built into the vast majority of models of formation and evolution of galactic disks over the past few decades (e.g. Tinslev 1975; Matteucci \& Francois 1989; Carigi 1996; Chiappini et al. 1997; Boissier \& Prantzos 1999).

Recent theoretical and observational evidence challenges this static picture. Spirals have long been known to be an important source of kinematic heating in galactic disks, gradually increasing the eccentricities of stellar orbits (Jenkins \& Binnev 1990). However, if a star is caught in the corotation resonance of a transient spiral it may move inward or outward in radius while preserv-

\footnotetext{
1 Astronomy Department, University of Washington, Box 351580, Seattle, WA 98195, USA roskar; trq@astro.washington.edu

${ }^{2}$ RCUK Fellow at Centre for Astrophysics, University of Central Lancashire, Preston, PR1 2HE, UK vpdebattista@uclan.ac.uk

${ }^{3}$ Department of Physics and Astronomy, McMaster University, Hamilton, ON, L8S 4M1, Canada stinson; wadsley@mcmaster.ca
}

ing the circularity of its orbit (Sellwood \& Binney 2002, hereafter SB02). Transient spirals with a wide range of pattern speeds are present throughout the evolution of the disk. Different pattern speeds result in spatially distinct locations of corotation resonances, allowing a star to undergo a series of resonant encounters in its lifetime, riding the spiral waves across large portions of the galaxy while retaining a mostly circular orbit. Stars born insitu may therefore remain kinematically indistinguishable from those that have come across the galaxy.

In Roškar et al. (2008) we presented first results from our $N$-body Smooth Particle Hydrodynamics (SPH) simulations of disk galaxy formation in which stars migrated radially due to their resonant interactions with transient spirals. These migrations yielded radial stellar population gradient predictions, which have recently been observed in resolved-star de Jong et al. 2007), and integrated-light studies (Azzollini et al. 2008; Bakos et al. 2008). In conjunction with our models, these observational data strongly imply that radial migration is an important process in observed galactic disks. In this Letter, we present further analysis of the simulation presented in Roškar et al. (2008), focusing on the wideranging implications of radial migrations for the observable properties of stellar populations.

\section{SIMULATION}

The initial conditions are created as in Kaufmann et al. (2006), and are designed to mimic the quiescent stage following a last major merger when thin disk formation commences. The system consists of a spherical dark matter NFW halo (Navarro et al. 1997) in which we embed a spherical halo of gas with the same initial profile. The gas halo is initially in hydrostatic equilibrium. The total mass of the system is $10^{12} \mathrm{M}_{\odot}$, analogous to a Milky Way-type spiral galaxy, with the baryons contributing $10 \%$ of the mass. We resolve the system with $10^{6}$ particles per component, resulting in initial mass resolutions of $10^{6} \mathrm{M}_{\odot}$ and $10^{5} \mathrm{M}_{\odot}$ for dark matter and gas respectively. Star 
particles form with masses that are a fraction of the gas particle mass, resulting in typical star masses around $3 \times 10^{4} \mathrm{M}_{\odot}$. To form a rotationally-supported disk, we also impart angular momentum to the gas component corresponding to a spin parameter value $\lambda=0.039$, as motivated by cosmological $N$-body experiments (e.g. Bullock et al. 2001). We evolve the system using the $N$-body + Smooth Particle Hydrodynamics code GASOLINE (Wadsley et al. 2004) for 10 Gyr.

Once the simulation begins, the gas cools and collapses to the center of the halo, forming a thin rotating disk from the inside-out. When the gas reaches densities and temperatures which allow for star formation, the star formation and supernova (SN) feedback cycles are initiated (Stinson et al.2006). Since our disks form without any $a$ priori assumptions about the interstellar medium (ISM) or the stellar populations present in the disk, we can self-consistently follow the evolution of their properties as the disk grows. Transient spirals cause radial redistribution of stellar material in a manner analogous to the mechanism presented in SB02. Due to space limitations, a detailed discussion of these processes is deferred until a future paper.

Our simulations do not account for the effects of evolution in a full cosmological context, but in the standard $\Lambda \mathrm{CDM}$ paradigm mergers are significantly more important at early epochs. The simulation presented here is therefore designed to mimic the quiescent phase of disk galaxy formation during which the thin disk forms. The increased resolution gained by stepping outside of the cosmological context allows us to isolate the effects of key dynamical processes, which determine multiple observational characteristics. Despite these simplifications, physical properties of our model such as the rotation curve, star formation rate, disk scale-length, and disk mass fraction, are analogous to those of observed systems. Pre-enrichment of merger fragments would not change the details of disk build-up, but simply offset the metallicity distribution.

\section{RADIAL MIGRATION AND ITS IMPLICATIONS}

Models of galactic chemical evolution have been enormously successful in explaining the properties of stars in our solar neighborhood (Matteucci \& Francois 1989; Carigi 1996; Chiappini et al. 1997; Boissier \& Prantzos 1999). One example of such model results is the agemetallicity relationship (AMR). The AMR is expected to arise due to progressive enrichment of the ISM through stellar feedback. Stars of the same age in the same general region of the galaxy are therefore expected to have similar metallicities. Indeed, early determinations of the AMR confirmed that the mean trend of stars in the solar neighborhood is toward lower metallicity with increasing age (Twarog 1980). Models, which assume that stars remain where they are born and return their nucleosynthetic yields to their local ISM, typically successfully reproduce this trend. However, evidence suggests that a large amount of scatter is present in the AMR of field stars (Edvardsson et al. 1993; Nordström et al. 2004) and open clusters (Friel et al. 2002), meaning that stars of the same age are observed to have a wide range of metallicities. In the framework of a galactic disk where radial annuli are self-enriched, homogeneous, and isolated from one another, old metal-rich stars or very young metal-poor stars are an impossibility.

Allowing stars from slightly different regions of the disk to enter the local sample due to the eccentricity of stellar orbits has been considered as a possible explanation, but can account only for up to $50 \%$ of the observed scatter (Nordström et al. 2004; Binney 2007). The large amount of scatter in the AMR therefore poses an important challenge for models of disk formation and should be considered as large a constraint as the mean trend (Carraro et al. 1998). The unexpected degree of scatter implies that stars in the solar neighborhood were either formed from a highly inhomogeneous ISM or have come from wildly different parts of the galaxy.

In our simulation, the latter option offers an enticing solution. In the left-most panel of Figure 1 we show the distribution of birth radii for stars, which at the end of the simulation are on nearly circular orbits within an annulus analogous to the solar radius. We define 'solar radius' as a general region of the disk interior to the disk break, but approximately 2-3 scalelengths from the center. In our simulated galaxy this region is between 7-9 kpc (indicated by dashed lines in Figure 11). The black line represents all stars while the blue and red lines show the distributions of metal poor $([\mathrm{Fe} / \mathrm{H}]<-0.3)$ and metal rich $([\mathrm{Fe} / \mathrm{H}]>-0.1)$ stars respectively. Roughly $50 \%$ of all "solar neighborhood" stars have come from elsewhere, primarily from the disk interior. Interestingly, some metal poor stars have been scattered into the solar neighborhood from the outer part of the disk. Such migration has recently been inferred from observational data (Haywood 2008). Metal-rich stars, like our Sun, could have originated almost anywhere in the Galaxy.

\subsection{Age-Metallicity Relationship}

It has previously been suggested that such large radial migrations could account for the scatter in the observed AMR (SB02, Binney 2007), but our models allow us to examine the implications of migrations in a self-consistent simulation of a growing and star-forming galactic disk. We show the AMR as determined from our simulation in the middle panel of Figure 1. Colors indicate relative particle densities for clarity in high-density regions. Diamonds and error bars indicate the means and standard deviations in several broad age bins. With the exception of the oldest age bin, the AMR is fairly flat. The relatively high number of very old ( $>9$ Gyr) extremely metal-poor stars is partly a remnant of our initial configuration, which includes only pristine, metalfree gas. This is likely to be different in a cosmological setting where a fraction of the gas is accreted from subhaloes that have already formed stars and been enriched by stellar feedback. Also, note that we assume each star is born with the mean metallicity of the ISM at its birth radius so that we can more easily isolate the effects of radial migration on the various observational relations.

To illustrate the impact of the dynamical evolution of the disk, we plot the AMR in these same age bins by using only stars that actually formed in the solar neighborhood. This latter relation is analogous to that derived by models which assume a dynamically non-evolving disk, essentially only depending on the nucleosynthetic yields and star formation and gas infall rates at the solar circle. In comparison to the full simulation, the in-situ relation shows a much tighter correlation between metal- 

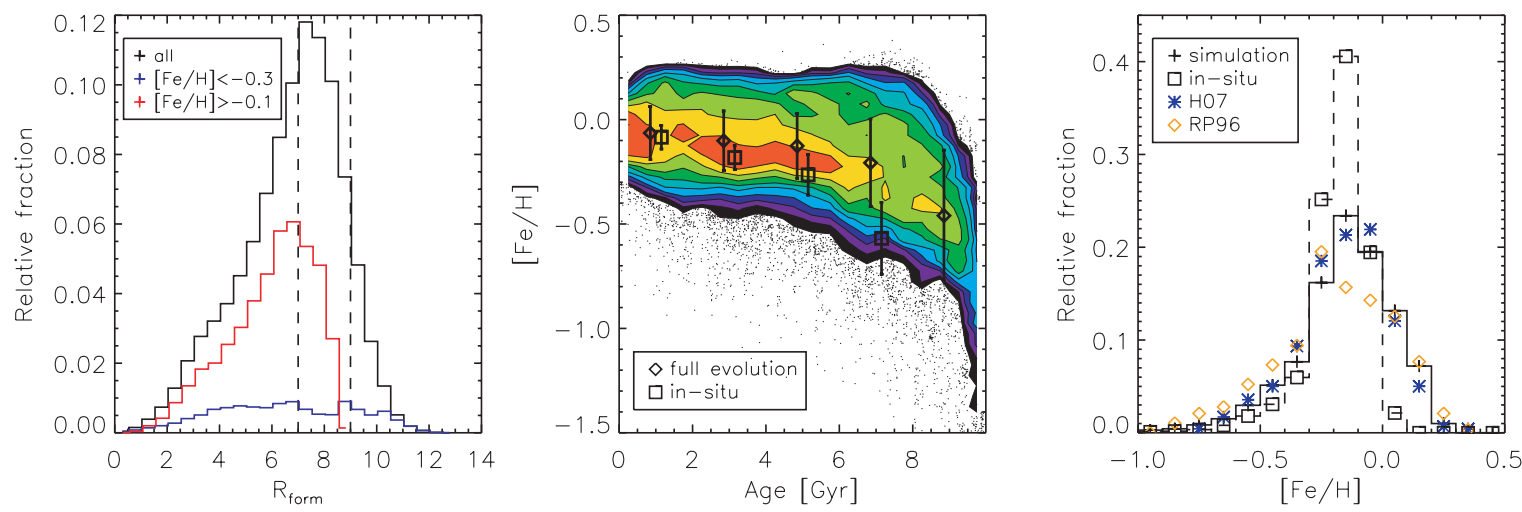

FIG. 1. - Properties of stars in the solar neighborhood. Left: Histogram of birth radii for stars that end up in the solar neighborhood on nearly circular orbits. The black, red, and blue lines represent all, metal-rich, and metal-poor stars respectively. Middle: The agemetallicity relation: color contours represent relative particle densities where point density is high. Diamonds and error bars indicate mean values and dispersion respectively. Squares show the AMR if stars are assumed to remain in-situ. A small horizontal offset is applied to the two sets of symbols for clarity. Right: Metallicity distribution function (MDF): the simulated distribution is shown with the solid black histogram; diamonds and asterisks show data from Rocha-Pinto \& Maciel (1996) and Holmberg et al. (2007) respectively. The dashed histogram is the MDF if stars are assumed to remain in-situ.

licity and age. Inclusion of full dynamical modeling significantly flattens the AMR and increases its dispersion by at least a factor of two. We note that although our simulations are not designed to reproduce Milky Way observables, they successfully reproduce the major qualitative features of the observations: the observed AMR is indeed relatively flat with a high degree of scatter (Edvardsson et al. 1993; Nordström et al. 2004), which gradually increases with age (Haywood 2008). This agreement strongly suggests that a large amount of radial migration is the missing piece of the AMR puzzle.

\subsection{Metallicity Distribution Function}

If radial migration is important for the AMR, then it should also leave a significant imprint on the metallicity distribution function (MDF), another key observational constraint for models of Galactic chemical evolution. The simple, closed-box chemical evolution picture where no material is allowed to enter or leave a given radial annulus has long been recognized as inadequate at explaining the local MDF (van den Bergh 1962). However, the dearth in the relative number of low-metallicity stars, known as the G-dwarf problem, is usually explained by allowing for the inflow of metal-poor gas (Larson 1974; Lvnden-Bell 1975). Gas infall "solves" the G-dwarf problem because it allows for prolonged star formation, which results in a relatively high number of metal-rich stars.

Our simulated MDF is shown with diamonds in the right panel of Figure 1 compared to observational data from Rocha-Pinto \& Maciel (1996) and Holmberg et al. (2007), represented by diamonds and asterisks respectively. ${ }^{4}$ We also plot the MDF of stars formed only in the solar neighborhood (shown with squares). Compared to the observed MDF, the in-situ distribution is much narrower. Radial migration introduces metal-poor and metal-rich stars into the solar neighborhood from other parts of the disk, thereby broadening the MDF.

Unlike the solar neighborhood AMR, which is highly dispersed and therefore difficult to use as a robust guide

4 Note that to shift the model quantities into the metallicity range relevant for the Milky Way, we apply an offset of +0.2 dex to the simulated metallicities. We show the observed distributions not to claim quantitative agreement, but simply to illustrate the ramifications of dynamical effects.

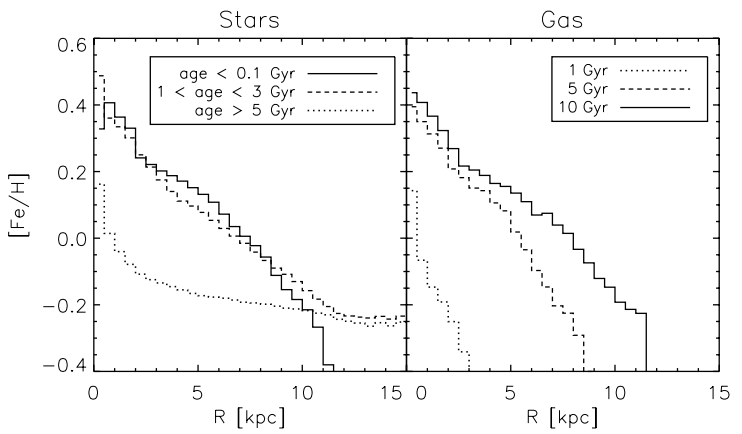

FIG. 2.- Left: Azimuthally averaged metallicity profile at 10 Gyr for stars in different age bins (young, intermediate age, and old stars shown with solid, dashed, and dotted lines respectively). Right: Azimuthally averaged metallicity profile of the gas at different times in the simulation.

for Galactic chemical evolution models, the MDF represents a much more stringent constraint. It is clear from Figure 1 that radial migration may significantly alter the nature of this fundamental property of the solar neighborhood.

\subsection{Metallicity Gradients}

A third major constraint on models of disk formation and evolution is the radial metallicity gradient. If stars are assumed to remain in-situ, then stellar tracers of different ages should provide information about the ISM metallicity from which they spawned. In the left-hand panel of Figure 2 we show the metallicity gradient as determined from stellar tracers of different ages. The slope of the gradient decreases with increasing age of stellar population. In most observational studies, such a trend would be interpreted to mean that the slope of the ISM metallicity was shallower in the past (Maciel et al. 2005). In the right-hand panel of Figure 2 we show the actual metallicity gradient in the ISM at different times in the simulation. Rather than being flatter in the past, as implied by the flat gradient of old stars, the ISM gradient was actually steeper and evolved in the opposite sense to that indicated by the stellar tracers. Radial migration causes more mixing in older populations, creating the appearance of flatter gradients at early times and leading to a decoupling of stellar properties from their birth ISM. 


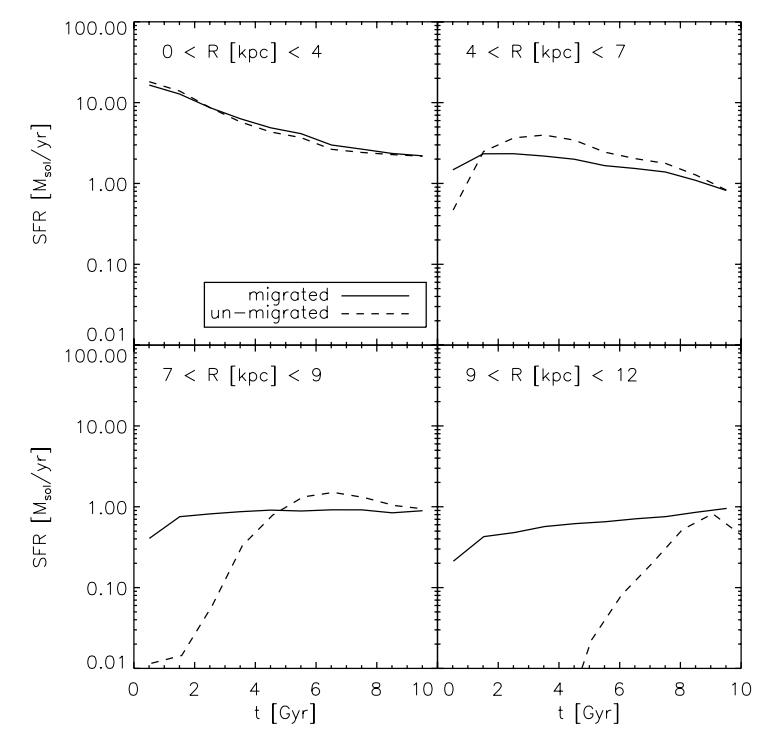

FIG. 3.- Star formation history in several broad radial bins.

\subsection{Star Formation History}

Implications of radial migration can be extended beyond our own Galaxy to extragalactic studies. In recent years, state-of-the-art observations of resolved stellar populations have begun to probe detailed, spatiallyresolved star formation histories in disks of external galaxies (e.g. Barker et al. 2007; de Jong et al. 2007; Williams et al. 2008; Gogarten et al. 2008). Typically, observed properties of stars in a given region of a disk are used to construct a color-magnitude diagram, which in turn is used to constrain stellar population synthesis models to yield a best-fit star formation history and therefore provide information about the formation of the disk (Dolphin 2002). Again, the underlying assumption is that stars observed today are found close to the radius of their respective birthplaces.

In Figure 3 we show the 'migrated' (solid) and 'unmigrated' (dashed) star formation history ( $\mathrm{SFH}$ ) in our simulation for several broad radial bins. The 'migrated' $\mathrm{SFH}$ is constructed by considering the distribution of ages in the desired region of the disk at the final snapshot of the simulation, much like an observer would do given a snapshot of a present day galactic disk. The 'un-migrated' SFH is the actual SFH, recovered by considering the times of formation for all stars formed within a given radial bin, regardless of the final radius to which they eventually migrated. We show here all stars regardless of the eccentricity of their orbits, but our conclusions remain the same even if we restrict our analysis only to stars on nearly circular orbits.

The discrepancy between the 'migrated' and 'unmigrated' SFH is large, especially for the outermost regions of the disk. For example, the 'solar radius' bin (7 $9 \mathrm{kpc}$ ) could erroneously be deduced to have a flat SFR for the past $\sim 8 \mathrm{Gyr}$, when it actually didn't reach current SFR levels until just $\sim 5$ Gyr ago. Migration must therefore be accounted for even in extragalactic studies of stellar populations.

\section{CONCLUSIONS}

We have shown that radial migration caused by resonant interactions of stars with transient spirals has significant repercussions on the observed properties of a wide range of stellar population systems. The range of implications and possible solutions to outstanding problems given by radial migration is appealing. Further work is required to characterize fully the degrees and timescales of migration in spiral galaxies of different physical characteristics. On the other hand, future observational efforts such as RAVE, GAIA and LSST will provide intricate data sets that should further constrain the radial migration process in the Milky Way. Ongoing and future HST projects such as the ANGST and GHOSTS surveys, will require radial migration models in order to reconstruct the $\mathrm{SFH}$ from resolved-star observations of nearby spiral disks and thereby learn about the formation history of our galactic neighborhood.

We would like to thank T. Kaufmann for the use of his initial conditions code. R.R. thanks C. Brook, J. Dalcanton and B. Gibson for useful discussions. This research was supported in part by the NSF through TeraGrid resources provided by TACC and PSC. R. R. and T. R. Q. were supported by the NSF ITR grant PHY-0205413 at the University of Washington. V. P. D. was supported by an RCUK Fellowship at the University of Central Lancashire. R. R. acknowledges support for a visit to the University of Central Lancashire from a Livesey Award Grant held by V. P. D.

\section{REFERENCES}

Azzollini, R., Trujillo, I., \& Beckman, J. E. 2008, ApJ, 679, L69 Bakos, J., Trujillo, I., \& Pohlen, M. 2008, ArXiv e-prints, 807 Barker, M. K., Sarajedini, A., Geisler, D., Harding, P., \& Schommer, R. 2007, AJ, 133, 1138

Binney, J. 2007, Dynamics of Disks (Island Universes - Structure and Evolution of Disk Galaxies), 67-+

Boissier, S. \& Prantzos, N. 1999, MNRAS, 307, 857

Brook, C. B., Kawata, D., Gibson, B. K., \& Freeman, K. C. 2004, ApJ, 612, 894

Bullock, J. S., Dekel, A., Kolatt, T. S., Kravtsov, A. V., Klypin, A. A., Porciani, C., \& Primack, J. R. 2001, ApJ, 555, 240 Carigi, L. 1996, RevMexAA, 32, 179

Carraro, G., Ng, Y. K., \& Portinari, L. 1998, MNRAS, 296, 1045 Chiappini, C., Matteucci, F., \& Gratton, R. 1997, ApJ, 477, 765 de Jong et al. 2007, ApJ, 667, L49

Dolphin, A. E. 2002, MNRAS, 332, 91
Edvardsson, B., Andersen, J., Gustafsson, B., Lambert, D. L.,

Nissen, P. E., \& Tomkin, J. 1993, A\&A, 275, 101

Friel et al. 2002, AJ, 124, 2693

Gogarten et al. 2008, ApJ, submitted

Haywood, M. 2008, ArXiv e-prints, 805

Holmberg, J., Nordström, B., \& Andersen, J. 2007, A\&A, 475, 519

Jenkins, A. \& Binney, J. 1990, MNRAS, 245, 305

Kaufmann, T., Mayer, L., Wadsley, J., Stadel, J., \& Moore, B. 2006, MNRAS, 370, 1612

Larson, R. B. 1974, MNRAS, 166, 585

-. 1976, MNRAS, 176, 31

Lynden-Bell, D. 1975, Vistas in Astronomy, 19, 299

Maciel, W. J., Lago, L. G., \& Costa, R. D. D. 2005, A\&A, 433, 127

Matteucci, F. \& Francois, P. 1989, MNRAS, 239, 885

Muñoz-Mateos, J. C., Gil de Paz, A., Boissier, S., Zamorano, J., Jarrett, T., Gallego, J., \& Madore, B. F. 2007, ApJ, 658, 1006 
Navarro, J. F., Frenk, C. S., \& White, S. D. M. 1997, ApJ, 490, 493

Nordström et al. 2004, A\&A, 418, 989

Robertson, B., Bullock, J. S., Cox, T. J., Di Matteo, T.,

Hernquist, L., Springel, V., \& Yoshida, N. 2006, ApJ, 645, 986

Rocha-Pinto, H. J. \& Maciel, W. J. 1996, MNRAS, 279, 447

Roškar, R., Debattista, V. P., Stinson, G. S., Quinn, T. R. Kaufmann, T., \& Wadsley, J. 2008, ApJ, 675, L65

Sellwood, J. A. \& Binney, J. J. 2002, MNRAS, 336, 785

Stinson, G., Seth, A., Katz, N., Wadsley, J., Governato, F., \& Quinn, T. 2006, MNRAS, 373, 1074
Tinsley, B. M. 1975, ApJ, 197, 159

Twarog, B. A. 1980, ApJ, 242, 242

van den Bergh, S. 1962, AJ, 67, 486

Wadsley, J. W., Stadel, J., \& Quinn, T. 2004, New Astronomy, 9, 137

White, S. D. M. \& Frenk, C. S. 1991, ApJ, 379, 52

Williams et al. 2008, AJ, submitted 